\title{
Manufacturing Technology of Polytetrafluorethylene Fibre
}

\author{
Le Luo ${ }^{1, a}$ \\ ${ }^{1}$ Chongqing Vocational Institute of Engineering, Chongqing, 402260, China \\ ${ }^{\mathrm{a}}$ email
}

Keywords: Polytetrafluorethylene fibre, PTFE, Manufacturing technology

\begin{abstract}
Polytetrafluoroethylene fiber is one of the most commonly used raw materials in industry. At present, there are there the popular manufacturing methods of polytetrafluoroethylene fiber, which are carrier spinning method, extrusion spinning method and membrane spinning method. The paper elaborates the above three methods and discusses the properties of polytetrafluoroethylene fiber through the experiments to provide some references for the relative researchers.
\end{abstract}

\section{Concept and Features of Polytetrafluorethylene Fibre}

Dr. Plunkett Du Pont found that the waxy white powder which is not dissolved and melting in 1938. The white powder was the polytetrafluorethylene fibre (short for PTFE). In 1950 this material began large-scale industrial production. PTFE has excellent corrosion resistance, excellent heat resistance and low coefficient of friction, self-lubrication, flame retardant, waterproof properties, has been widely used in industrial fields of petrochemical industry, electronic and electrical, aerospace, semiconductor, bio pharmaceutical, textile. PFA contains only vinyl ether, significantly improved the flexibility of polymer chain of the PFA has good thermal plasticity, overcomes the shortcomings of PTFE hard machining. The long-term use of temperature and PTFE are the same, but the mechanical properties at high temperature are larger than the PTFE times.

Due to the special characteristics of PTFE molecular structure, compared with ordinary plastics, PTFE has many excellent qualities. PTFE has chemical stability. The C-F bond in PTFE molecule has very high bond energy. In addition to the strong fluoride medium, molten alkali metal and fluorine and 300 CENTIGRADE to the effect of some sodium hydroxide, all strong oxidizing and reducing agents, strong acid and strong alkali and organic solvent had no effect on the. Even in the boiling aqua will not affect its quality and performance. In addition to the above $300 \mathrm{~g} / \mathrm{g}$, the temperature is slightly higher than that of all the alkanes, which is almost insoluble in all the solvents in the ratio of about $0.1 \mathrm{~g} / 100 \mathrm{~g}$. PTFE does not burn, does not absorb moisture, and in the ultraviolet and oxygen environment are very stable, with excellent weather resistance. The maximum instantaneous operating temperature can reach 290 centigrade, and even under a low temperature of a temperature of 260 centigrade. The molecular chain of FFFE is a nonpolar molecular chain with good dielectric properties and excellent arc resistance. Even in the case of high voltage discharge, PTFE will only release a small amount of non-conducting gas, but it will not be short circuited. Because of the low molecular weight of PTFE, the molecular chain of PTFE is very small, and the PTFE molecular chain is a chain of high rigidity and high entanglement. Therefore, when the load is applied to the PTFE for a long time, the creep will occur, and it is easy to produce the phenomenon of cold flow, but its fatigue resistance is very good.

\section{Common manufacturing Methods of Polytetrafluorethylene Fibre}

Carrier Spinning Method. Some fiber difficult to use traditional spinning technology to simplify the process of preparation or coarse fiber needs the carrier and make hard into fiber forming polymer particles at the temperature above the melting point of adhesion under high temperature, the carbon residue which contains dark brown. The most difficult to make polymer fibers are emulsion or powder with less than three nm diameter, and the carrier can be blended uniformly with emulsion or 
micro powder. The carrier is the main method for producing PTFE fiber spinning industry, is generally PFTE emulsion with viscose or polyvinyl alcohol aqueous solution into a fibrous carrier mixture made of spinning liquid, spinning liquid through the filter into the spinning head by wet spinning. The spinning head is placed in the coagulating bath, such as sodium sulfate, and the mixture is condensed into a fiber in the coagulation bath through a spinning head. The formed fiber is sintered at high temperature after being washed and dried, and the carrier is carbonized to remove, and then the PTFE fiber which is formed by sintering is continuously stretched to obtain the finished fiber. At present, it can be used to produce fine staple fiber.

Emulsion spinning is a kind of carrier spinning. It is a kind of polymer material which is dispersed in a kind of spinning material, and then it is spun in the usual way. The carrier is commonly used in viscose or polyvinyl alcohol aqueous solution. The nascent fibers were sintered at high temperature after drawing, the carrier was carbonized, and the polymer particles were bonded to form fibers near the viscous flow temperature. The polymer fibers suitable for emulsion spinning should have a melting point higher than the decomposition temperature, and there is no suitable solvent to dissolve or plasticizing. It cannot be made into melt and spinning solution. At present, this method has been used in the production of PTFE fiber and so on.

Extrusion Spinning Method. The properties of spinning solution of PTFE fibre can be divided into paste extrusion spinning and gel extrusion spinning. Paste extrusion is usually spinning lubricating material PTFE powder and a volatile paste, the extrusion machine, in $15 \sim 20 \mathrm{MPa}$ through a spinneret spinning, then drying and sintering, the lubricant volatilization, after drawing non-white stripe yarn uniform, the fibres are not the exact size. However, these strips have finished fibre yarn processing PTFE. There is thin film or extrusion in the extrusion device, after rolling process, the removal of additives. Because it does not contain binder carbonization residue, this method to obtain the PTFE fibre strength is relatively high, usually made of thin film twisting belt, high tensile strength of woven yarn, sewing thread, monofilament, dust bag and joint sealant. Gel extrusion spinning is a kind of spinning and spinning process. It is usually PTFE concentration dispersion emulsion and polyvinyl alcohol mixed with polyvinyl alcohol under alkaline conditions and borate or borax gel forming characteristics of preparing gel spinning solution. The spinning liquid is transported to the spinning head by a gas pressure or screw, and then the dry spinning and drying are carried out. The obtained mixed fibre is made into polytetrafluoroethylene fibre. The polytetrafluoroethylene fibre prepared by the method has good quality and can be widely used in filtering materials.

Membrane Spinning Method. This method is mainly used to pull into thin strips after sintering drawing process. The existing pull cutting equipment cannot be cut into very fine material level, therefore making coarse fibres, uneven fineness. At the same time the method is used in bulk PTFE powder, high cost. This method made PTFE multifilament used as sealing filler material for short fibre needle felt. The method of membrane cracking spinning is to make the polymer into a thin film, and then the fibre is made by mechanical processing. According to the different processing methods, the fibre is divided into two kinds of broken fibre and tearing fibre. Split fibre is also called flat, the processing way is to cut into thin strips of a certain width, stretching several times, and be finished on the bobbin winding. Processing method of tearing fibre is the film along the longitudinal tension, the molecular orientation along the axial direction fully, also produce crystal, with chemical and physical methods make the structure relaxation, and the mechanical effect of torn into filaments, then twisting and curling finished. The former coarse fibre is used to instead the hemp as packing material. The latter is slightly thinner, used to make carpets and ropes. At present, it is used in the production of polypropylene fibre.

The non-stretched filaments obtained in the spinning process. Its structure is not perfect and stable, physical and mechanical properties are poor, is not suitable for textile processing. Therefore, it is necessary to go through the post-processing process. The process varies with the fibre varieties and types. Drawing and heat setting have a direct impact on the structure and properties of finished fibre. By using wet spinning fibres but also after washing, to remove the attachment of the coagulation bath fluid and solvent; the staple fibre to curl and cut production; the need for long fibre twisting and winding. These processes have little effect on the structure of the fibres, and thus have little effect on 
the properties of the fibres. Given the fibre with some special properties, such as wrinkle resistance, hot water resistance and rebound performance. Some special processing is needed in post-processing process.

\section{Performance Testing of Polytetrafluorethylene Fibre}

Thermal Gravimetric Analyzer. Thermal gravimetric analyzer is a kind of instrument to detect the change of temperature and mass of matter. The thermal gravimetric method is used to measure the relationship between the mass of the material and the temperature (or time). The most commonly used measurement of the principle of two kinds, that is the displacement method and zero method. The so-called displacement method is based on the balance beam tilt and mass changes in the proportion of the relationship between the uses of differential transformer. Zero method is the determination of the inclination of the balance beam by using differential transformer method and optical method, and then to adjust the installation in the balance system and current coil in a magnetic field, the coil rotation recovery tilt the balance beam, the so-called zero method. As the force applied to the coil is proportional to the mass change, the force is proportional to the current in the coil, so the curve of mass change can be obtained by measuring and recording the current. We call for 5 to $10 \mathrm{mg}$ PTFE samples were dried. It recorded the $\mathrm{N} 2$ and air $(40 \mathrm{ml} / \mathrm{min})$ heating rate from 50 degrees to 800 degrees. The curve (Fig. 1) presented the fact that PTFE has a good heat resistance nature. It won't dissolve until the temperature reaches 500.6 degrees.

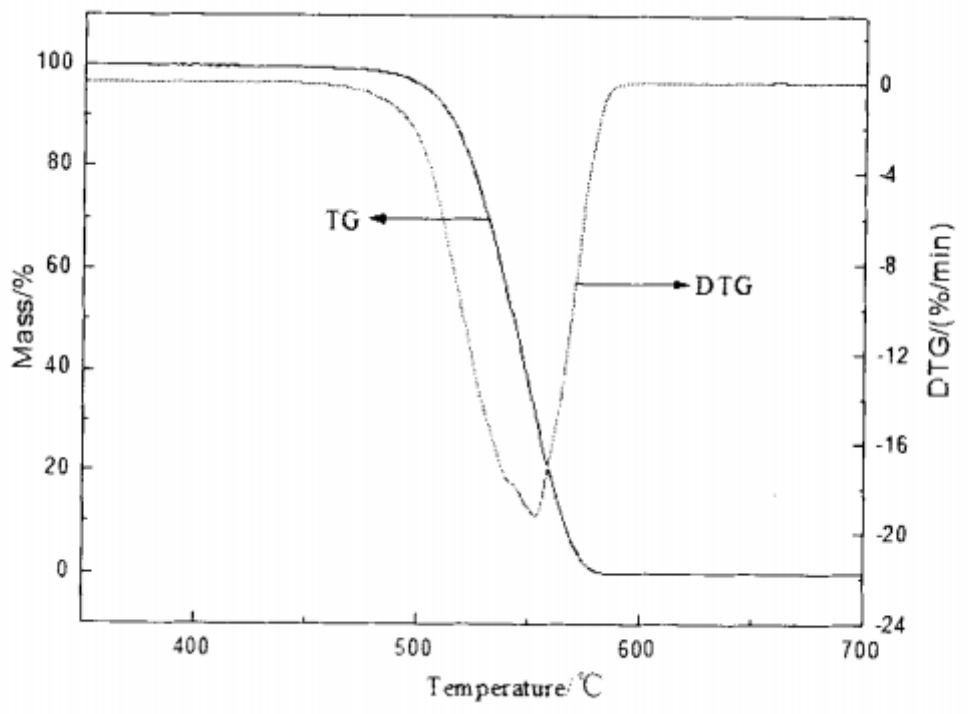

Fig. 1: TG curve and DTG curve of the Polytetrafluorethylene fibre

Infrared Spectra Analysis. When a beam of different wavelengths of infrared radiation is applied to the material, some specific wavelengths of infrared rays are absorbed to form the infrared absorption spectrum of the molecule. Each molecule has its own unique infrared absorption spectrum determined by its composition and structure. The infrared absorption spectrum is produced by the vibration and rotation of the molecule. The molecular vibration is the relative motion of each atom in the molecule. When the molecules atoms in the same frequency and phase near the equilibrium position of simple harmonic vibration, the vibration is called normal vibration. Infrared ray energy and molecular vibration of the photons corresponds to, so when the vibration state of the molecular changes, it can emit infrared spectrum, can be excited by infrared radiation of molecules vibrate infrared absorption spectrum. The molecular vibration and rotation energy is not continuous but quantized. However, in the process of the vibrational transition of molecules, it is often accompanied by the rotational transition, which makes the vibrational spectrum. So the infrared spectrum of the molecule belongs to the band spectrum. Figure 2 shows that the infrared spectrum of PTFE. 1154 cm-1 and 1209 cm-1 are the peaks of the C-F bond stretching vibration absorption. 


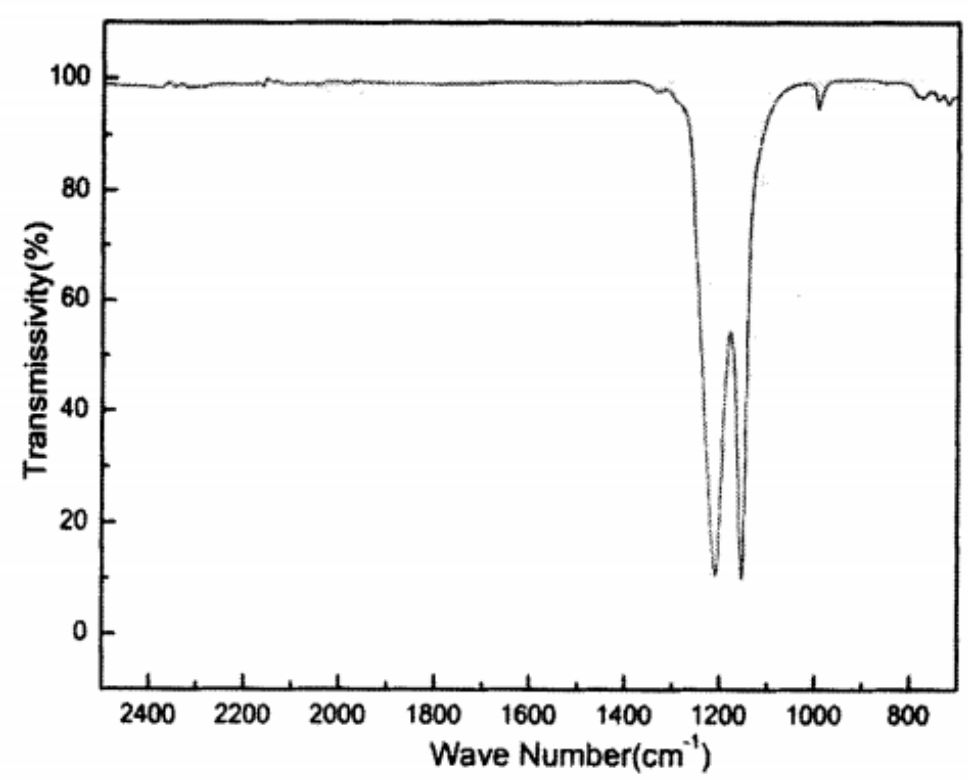

Fig. 2: Infrared spectra figure of Polytetrafluorethylene fibre

\section{Conclusion}

PTFE fibre has many excellent properties and has been widely used in aerospace, petrochemical, marine operations, textile, food, paper and other fields. At present, the PTFE spinning methods include the membrane spinning, extrusion spinning and carrier spinning. We believe that with the development of science and technology, PTFE fibre production methods will continue to progress. With the popularization of PTFE nano fibres, the application field of PTFE fibre will be more extensive.

\section{References}

[1] Chen Guanfushou, Huang Binxiang, Preparation and application of high performance polytetrafluoroethylene fiber scrim[J]. New Chemical Materials, 2013, 41(11): 185-186+195.

[2] Chen Liping, Wang Yaowu, Sun Runjun, Chen Meiyu, Preparation and Characterization of PTFE Fiber[J]. Synthetic Fiber in China, 2010 (10): 24-26+34.

[3] Zhou Ming, Zhang Haofan, Song Shuang, Chen Wenqing, Ar Plasma in Hydrophilic Modification of PTFE Hollow Fiber Membrane[J]. Guangzhou Chemical Industry, 2016, 44(12): 84-86.

[4] Wang Jinghong, Diao Yongfa, Experimental study on adsorption capability of PTFE fiber cooperated with magnetic adsorbent for Hg0removal in coal-fired flue gas[J]. New Chemical Materials, 2016, 44(12): 147-149. 